\title{
El papel del tercer sector de la región fronteriza hispano- marroquí en la situación de las mujeres porteadoras en Ceuta.
}

The role of the third sector of the Spanish-Moroccan border region in the situation of 'carrier' women in Ceuta.

\section{Cristina FUENTES LARA}

Universidad Rey Juan Carlos/Universidad Francisco de Vitoria ${ }^{1}$

cristina.fuentes@urjc.es

http://orcid.org/0000-0003-3198-7701

Recibido 29/8/2018. Revisado y aceptado para publicación 28/2/2019

Para citar este artículo: M. Cristina FUENTES LARA (2019), "El papel del tercer sector de la región fronteriza hispano-marroquí en la situación de las mujeres porteadoras en Ceuta" en Revista de Estudios Internacionales Mediterráneos, 26, pp. 144-159.

Para acceder a este artículo: https://doi.org/10.15366/reim2019.26.009

\begin{abstract}
'Carrier' are Moroccan women who work in Ceuta carrying bales of 50 kilos between the two sides of the Spanish-Moroccan border of Ceuta. This work is carried out by women porters in exchange for a small economic commission for the displacement carried out and the activity carried out. The porters develop their work activity in very poor conditions where their human rights are systematically violated, while the rest of the agents involved in the atypical trade obtain important economic benefits with this activity. The methodology that has been followed in this investigation has been the participant observation and the in-depth interview with the social agents and carrier women; while the technique of data analysis has been the Grounded Theory. The results of the investigation show the tendency that although the third sector - unions, associations, collectives is motivated to intervene in the situation of porters, they do not find the appropriate methods or actions to dignify the situation of women porters in the Spanish-Moroccan border.
\end{abstract}

Keywords: 'carrier' women/ border/Third sector/ atypical trade/women.

\footnotetext{
${ }^{1}$ Profesora asociada de Ciencias de Comunicación y Sociología. Universidad Rey Juan Carlos. investigadora de la Cátedra de Inmigración, Universidad Francisco de Vitoria.
} 


\section{Resumen}

Las porteadoras son mujeres marroquíes que trabajan en Ceuta cargando con fardos de 50 kilos entre los dos lados de la frontera hispano-marroquí de Ceuta. Este trabajo lo desempeñan las mujeres porteadoras a cambio de una pequeña comisión económica por el desplazamiento realizado y la actividad desempeñada. Las porteadoras desarrollan su actividad laboral en unas condiciones paupérrimas donde sus derechos humanos son sistemáticamente vulnerados, mientras que el resto de agentes implicados en el comercio atípico consiguen importantes beneficios económicos con esta actividad. La metodología que se ha seguido en esta investigación ha sido la observación participante en los puntos clave de la investigación y la entrevista en profundidad a los agentes sociales y a las mujeres porteadoras. En cuanto a la técnica de análisis de datos empleada en esta investigación ha sido la teoría fundamentada (Grounded Theory). Los resultados de la investigación muestran la tendencia de que si bien el tercer sector -sindicatos, asociaciones, colectivos- se encuentra motivado para intervenir en la situación de las porteadoras no encuentran los métodos ni las acciones adecuadas para dignificar la situación de las mujeres porteadoras en la frontera hispano-marroquí.

Palabras clave: porteadoras/ frontera/tercer sector/comercio atípico mujeres.

\section{Introduction}

Las porteadoras ${ }^{2}$ son mujeres marroquíes principalmente residentes en los municipios de la wilaya de Tetuán, ${ }^{3}$ que se desplazan diariamente a Ceuta para trabajar cargando con fardos de mercancía desde el polígono del Tarajal (Ceuta) hasta Tora Bora o parking (lado marroquí de la frontera) a cambio de una comisión económica de unos 20 euros por día, aproximadamente (Fuentes-Lara, 2017b). El fardo con el que cargan durante horas puede llegar a pesar 50 kilos y contiene productos de alimentación, higiene y limpieza, tecnología, calzado, ropa y mantas (Fuentes-Lara, 2016; Ferrer-Gallardo, 2008). El desempeño de su trabajo lo realizan en unas condiciones sociolaborales nefastas donde no son reconocidas como trabajadoras, sino que son consideradas como individuos que se desplazan a Ceuta a realizar compras para su hogar a título individual. Las porteadoras tienen que esperar horas a la intemperie, sin agua potable ni baños públicos, expuestas a la violencia estructural, física y sexual de la frontera hispano-marroquí, lo que supone una flagrante violación de sus derechos humanos (Rosado-Caro y Fuentes-Lara, 2018).

Las condiciones de cruce de la frontera hispano-marroquí es cambiante y fluctúa en función de los múltiples actores políticos y sociales que interactúan en la zona fronteriza, tales como la Unión Europea, España, Marruecos, los gobiernos locales de los municipios fronterizos, los comerciantes del polígono del Tarajal, los clientes de la mercancía etc. Eso sí, cada uno de ellos con un peso diferenciado dentro de la estructura de poder de la zona fronteriza. Ejemplo de ello, es la decisión unilateral del Reino de Marruecos de cerrar la aduana comercial con Melilla el 31 de julio de 2018, pero no es la única medida reciente que influye en las porteadoras. Los comerciantes del Tarajal en mayo de 2018 decidieron, sin consensuar con el resto de agentes, implantar el uso de "carritos de compra" para sacar la mercancía del polígono del Tarajal (Rosado-Caro y Fuentes-Lara, 2018).

\footnotetext{
${ }^{2}$ En este artículo se va a abordar únicamente la situación de las mujeres porteadoras en la frontera hispano-marroquí de Ceuta. Aunque el caso de las mujeres porteadoras en Melilla presenta similitudes con el de Ceuta, para esta investigación se ha optado por centrarse en la zona fronteriza ceutí.

${ }^{3}$ El termino de wilaya en árabe puede ser traducido como región. Concretamente la wilaya de Tetuán engloba los municipios de M'diq (Rincón), Fnideq (Castillejos), Martil y Tetuán. Un área de 40 kilómetros.
}

REIM № 26 (junio 2019) 
Pese a que son dos ejemplos de distinta índole jurídico-política, ambos casos representan la toma de decisiones en el trasiego de mercancías entre las Ciudades Autónomas y Marruecos, en el que las mujeres porteadoras no son consultadas ni informadas.

Las mujeres porteadoras se encuentran en el limbo jurídico, ya que carecen de un articulado legislativo que regule o prohíba su trabajo(Ferrer-Gallardo y Albet-Mas, 2016) y también dentro de la toma de decisiones relativas a su actividad laboral (Fuentes-Lara, 2018). Si bien se ha estudiado extensamente la cuestión de las mujeres porteadoras (Fuentes-Lara, 2016, 2017a, 2017b; Planet, 1998; Ferrer-Gallardo, 2008; Espiñeira, 2013; Saceda 2016; Galán, 2012), no se ha prestado suficiente interés en los agentes que perpetúan el circuito de las mujeres porteadoras (FuentesLara, 2017b) ni al papel del tercer sector -entendido como las asociaciones, los sindicatos, los colectivos sociales y el voluntariado que no realicen actividades laborales con ánimo de lucro (Pérez-Díaz y López-Novo, 2003)- en la defensa de la vulneración de derechos humanos de las mujeres porteadoras.

Precisamente, es de esta motivación de la que radica el interés de este artículo científico, cuyo objetivo es conocer el papel del tercer sector en la situación de las mujeres porteadoras en Ceuta. Los objetivos específicos de esta investigación son indagar sobre las características y presencia del tercer sector en la zona fronteriza; estudiar las problemáticas que tiene el tercer sector para la intervención con porteadoras; y examinar la confianza entre porteadoras y tercer sector. En este artículo se sigue la siguiente estructura, en primer lugar se realizará una contextualización teórica en la que se detallan los condicionantes que favorecen la actividad laboral de las mujeres porteadoras; a continuación se expone la metodología que se ha seguido en la investigación. Por último, se presentan los resultados de esta investigación, y para finalizar, se llegaran a las conclusiones principales de este artículo.

\section{Contextualización}

La actividad laboral de las mujeres porteadoras se ve favorecida por la singularidad de la zona fronteriza de la Ciudad Autónoma de Ceuta. En primer lugar, respecto a las características geográficas de Ceuta ya que junto a Melilla son los únicos territorios europeos en el continente africano (López-Bueno, 2008). Por ello el Reino de Marruecos no reconoce a ambas Ciudades Autónomas como territorios españoles ni acepta la existencia de fronteras entre los límites de las ciudades y Marruecos (García-Flórez, 1999).

En segundo lugar, y relacionado con lo anterior, Ceuta cuenta con una singularidad fiscal y legislativa. Respecto a la fiscal, desde la aprobación del Real Decreto del 13 de enero de 1860, Ceuta es declarada puerto franco, o sea, que disfruta del privilegio de franquicia de derechos de aduana sobre los bienes y las mercancías que entren a la Ciudad Autónoma (Muñoz, 1986) que únicamente pueden introducirse por vía marítima por el puerto de Ceuta. Las mercancías que entran a Ceuta solamente son gravadas por un impuesto de recaudación municipal, el Impuesto sobre la Producción, los Servicios y la Importación (IPSI). EI IPSI es un impuesto el equivalente al Impuesto sobre el Valor Añadido, con la diferencia de que tiene una tasa impositiva inferior a la del IVA, dado que grava los bienes del 0,5\% al 10\% (González y López-Guzmán, 2009). En cuanto a la singularidad legislativa, Ceuta no forma parte de la Unión Aduanera Comunitaria, a petición propia, con la finalidad de no perder sus beneficios fiscales (Muñoz, 1986), y goza de un estatus especial en el Acuerdo Schengen que determina su relación con el entorno marroquí.

El Acuerdo Schengen fue firmado por España en 1991. En la Ratificación el Protocolo de Adhesión de España al Acuerdo Schengen se incluyó una declaración sobre Ceuta y Melilla, en la que se señala que "el régimen específico de exención de visado en materia de pequeño tráfico fronterizo 
entre Ceuta y Melilla y las provincias marroquíes de Tetuán y Nador" (BOE, 1994: 3). Por consiguiente, la población marroquí que tenga un pasaporte emitido en la wilaya de Tetuán o Nador, no necesita visado para entrar en las Ciudades Autónomas pero con unas limitaciones, ya que no puede desplazarse a la península y no puede pernoctar en las ciudades. La justificación radica en que pueden entrar a Ceuta o Melilla únicamente para trabajar o para realizar compras. Para autores como Ferrer-Gallardo (2008), Espiñeira (2013) y Moffette (2013) esto responde a una política de filtrajes o una 'schengenización' selectiva de Ceuta y Melilla.

En tercer lugar, la inexistencia de una aduana comercial entre Ceuta y Marruecos. La frontera del Tarajal es un paso fronterizo para personas no para mercancías (Fuentes-Lara, 2016) puesto que Marruecos no reconoce la existencia de una frontera (Ferrer-Gallardo, 2008). Por ello no se pueden realizar exportaciones regulares entre ambos lados de la frontera, aunque como señala Saceda (2016) el flujo de bienes y mercancías de Ceuta a Marruecos en un hecho cotidiano. Eso sí, por medio de la actividad de las mujeres porteadoras, la cual se ve amparada por la legislación marroquí, dado que el Decreto-ley número 1-77-339 del 9 de octubre de 1977 que regula la entrada de bienes a Marruecos no precisa los límites de lo que se considera como contrabando (Planet, 1998), y es más, afirma que una persona puede introducir bienes en su territorio siempre y cuando los lleve encima de cuerpo, sin especificar peso ni cantidad permitida.

La situación descrita ampara el llamado "comercio atípico" entre ambos lados de la frontera ceutí. Esta circunstancia genera amplios beneficios económicos a los comerciantes del polígono del Tarajal, a los clientes de la mercancía del lado marroquí y a la Ciudad Autónoma. De hecho, Carabaza y Santos (1993) aseguran que España conserva la soberanía de Ceuta y Melilla por los beneficios del contrabando con Marruecos, postura que de forma más moderada apoyan FerrerGallardo (2008) y García-Flórez (1999) para quienes el comercio atípico es un pilar básico en el sustento económico de Ceuta.

Respecto a la recaudación económica o los beneficios económicos que la actividad de las porteadoras genera a la Ciudad Autonomía, se pueden estudiar en función de la recaudación de impuestos y la dinamización empresarial de la ciudad. En cuanto al primero de ellos, hay que destacar como el $25 \%$ de los ingresos de la Ciudad Autónoma provienen de la recaudación del Impuesto sobre la Producción, los Servicios y la Importación (IPSI) a la importación (Presupuesto de Ceuta, 2017). En el mismo año 2017, la recaudación del IPSI a la importación llegó a los 65.756.000 € anuales, una cifra que sería impensable sin la existencia del comercio atípico, dado que en Ceuta no existe agricultura y no hay un desarrollo industrial, únicamente cuenta con el hiperdesarrollado sector servicios instalado en el polígono del Tarajal. En el análisis del polígono del Tarajal se encuentra el entramado del comercio atípico. El Tarajal es un polígono comercial de concesión privada que aglutina 286 naves comerciales dedicadas a la venta mayorista según la clasificación del CNAE ${ }^{4}$. El polígono se encuentra ubicado al lado de la frontera de El Tarajal, alejado del centro más comercial de Ceuta, lo cual manifiesta que la funcionalidad del polígono no es el comercio con la población ceutí si no favorecer el comercio atípico.

El enclave geográfico ceutí obliga a que la llegada de mercancías desde fuera de la ciudad se realice únicamente por vía marítima por medio del puerto de Ceuta. Pues bien, en la última memoria publicada por la Autoridad Portuaria de Ceuta en 2015, se contabilizan 154 toneladas de mercancías alimenticias, un 0,03\% del total de exportaciones de ese año (2015), una cifra muy

\footnotetext{
${ }^{4}$ De hecho, Ceuta cuenta el mayor número de establecimientos comerciales mayoristas por cada mil habitantes de España (INE, 2017).
}

REIM № 26 (junio 2019)

ISSN: $1887-4460$ 
superior al consumo alimenticio de una población de 84.519 habitantes. ${ }^{5}$ Estos datos evidencian cuál es la finalidad del polígono del Tarajal, que no es otra que el almacenamiento de bienes y de mercancías para su posterior exportación, no legalmente regulada a Marruecos, y justamente en bienes alimenticios que son uno de los productos que suelen cargar en los fardos las mujeres porteadoras (Fuentes-Lara, 2016).

De hecho, existe una postura generalizada que afirma que el volumen de transacciones del comercio atípico es notablemente superior al total de las exportaciones legales de España hacia Marruecos (Soddu, 2002; García-Flórez, 1999), mientras que Amirah (2013) en un estudio del Real Instituto Elcano, estima que el comercio atípico rondaría el 30\% de las exportaciones legales entre los dos países (2013: 2). Esto se traduciría en que en el año 2016, el comercio atípico en Ceuta y Melilla, generó exportaciones a Marruecos por valor de 1.702 .609 euros.

De tal forma que mientras que por un lado se obtienen cuantiosos beneficios económicos, por otro lado se esclaviza a unas mujeres con una compleja situación personal y económica. Toda esta paradoja de enriquecimiento de agentes fronterizos -Ceuta, comerciantes del Tarajal, clientes de la mercancía etc.-, mientras que se vulneran los derechos humanos de las mujeres porteadoras, se produce ante la pasividad del tejido de la zona fronteriza, que no interfiere vehementemente en la lucha para la defensa de los derechos de las porteadoras.

\section{Material y método}

La metodología de esta investigación es de corte cualitativo, puesto que la finalidad del método esta "orientada a descubrir, captar y comprender una teoría, una explicación o un significado" (Ruiz-Olabuénaga, 1996: 57), tal y como se ha dado en esta investigación. Las fuentes de información de la investigación han sido predominantemente primarias, aunque se han empleado datos secundarios cuantitativos para apoyar datos cualitativos o para contextualizar el objeto de la investigación. La fase de trabajo de campo se realizó entre mayo de 2015 y octubre de 2017.

Las técnicas de producción de datos utilizadas en esta investigación han sido la observación participante y la entrevista en profundidad. Para Velasco y Díaz de Rada (2006), estas técnicas son complementarías ya que captan "los productos y los modelos, los comportamientos y los pensamientos, las acciones y las normas, los hechos y las palabras, la realidad y el deseo" (p. 33). Si bien en la observación participante los datos se obtienen en el desarrollo de la acción de los sujetos objeto de la investigación, en la entrevista en profundidad, son los sujetos y demás informantes, son quienes detallan la información sobre la acción social (Fuentes-Lara, 2017c).

La observación participante se realizó en unos puntos concretos seleccionados por desarrollarse en ellos la acción social -es decir, el trasiego de mujeres porteadoras y la interacción entre porteadoras y agentes sociales- (Díaz de Rada, 2011). Los puntos de observación se dieron en ambos países y se atendió al criterio de flexibilidad. De tal forma que hubo puntos de observación en los que se cesó la actividad investigadora6. Los puntos donde la observación requirió de más repeticiones fueron: la frontera internacional del Tarajal (España y Marruecos), el polígono del Tarajal (Ceuta, España), Tora Bora o 'parking' (Marruecos), centro comercial de Ceuta (Ceuta, España) y Medina de Tetuán (Tetuán, Marruecos).

\footnotetext{
${ }^{5}$ Datos del padrón municipal a fecha de 1 de enero de 2017 extraídos del Instituto Nacional de Estadística (INE).

${ }^{6}$ Los zocos de las localidades de Fnideq, M’diq, Martil y Tánger tras realizar el análisis de la información recaba no se consideraron de relevancia para la investigación al no transcurrir en ellos la acción social.
} 
La entrevista en profundidad fue la técnica sobre la que recayó el mayor peso de la investigación, entendida como un "diálogo face to face, directo y espontáneo, de una cierta concentración e intensidad entre el entrevistado y un sociólogo más o menos experimentado, que oriente el discurso lógico y afectivo de la entrevista de forma más o menos directiva" (Ortí, 2010: 272). Las entrevistas fueron semiestructuradas, para lo cual se diseñó un guion de entrevista orientado a indagar sobre el posicionamiento de los agentes sociales sobre el fenómeno del comercio atípico y la situación de las mujeres porteadoras. El guion de entrevista se ajustaba a unos lineamientos generales y se iba adaptando en base al tipo de agente social que se entrevistaba, puesto que se entrevistaron diferentes perfiles, desde la confederación de empresarios, tejido asociativo, sindicatos de las fuerzas del orden y representantes de partidos políticos. En cuanto a la reformulación del guion de la entrevista, se fue realizando conforme se avanzaba en el proceso de saturación teórica, añadiendo o eliminando variable cuando alcanzaban la saturación.

La selección de las personas entrevistas informantes se realizó a partir de los listados de tercer sector de la wilaya de Tetuán y de Ceuta. ${ }^{7}$ En cuanto a las asociaciones y ONG's se seleccionaron aquellas que tenían temáticas afines al objeto de la investigación, o sea, derechos humanos y género. La primera de ellas, los derechos humanos, se considera un área cercana a esta investigación por las flagrantes violaciones a los derechos humanos que se producen en la situación de las mujeres porteadoras, tales como la ausencia de baños públicos, la inexistencia de fuentes de agua potable, ineficacia de zonas sombreadas, la violencia física y psicológica por parte de las fuerzas y cuerpos de ambos estados y comerciantes del Tarajal (APDHA, 2012; Rosado-Caro y Fuentes-Lara, 2018; Fuentes-Lara, 2018). Respecto a la implicación del tercer sector que trabaja en temas de género, su relación es más que directa puesto que en el circuito de las porteadoras radica una estructuración férrea de género. Si atendemos a la configuración del circuito de las porteadoras se refleja claramente cómo los agentes que se benefician del trabajo de las porteadoras son hombres -clientes marroquíes de la mercancía, comerciantes del Tarajal, agentes aduaneros, ${ }^{8}$ intermediarios entre porteadoras y comerciantes-, mientras que son ellas, las mujeres porteadoras las que realizan la actividad fundamental del comercio atípico y quienes sufren la vulneración de sus derechos humanos (Fuentes-Lara, 2017b).

Una vez detectadas las asociaciones de la zona fronteriza que desarrollaban acciones en temáticas similares a las porteadoras -género, frontera, derechos humanos-, o que se viesen afectadas por la situación de las mujeres porteadoras -asociaciones de comerciantes del Tarajal, sindicatos o asociaciones de los cuerpos y fuerzas de seguridad del Estado-, se contactó con ellas para explicar el objetivo de la investigación y solicitar una entrevista. En este punto aparecieron dos problemas. El primero, que del listado de tercer sector registrado en los Ayuntamientos, muchas de las asociaciones ya no tenían actividad, lo cual redujo la selección cuantiosamente. Y segundo, las asociaciones eran reticentes a fijar una entrevista. La explicación versaba en qué no trabajaban con mujeres porteadoras y por ende, no tenían información que aportar respecto a su trabajo o su situación -esto ocurrió especialmente en Ceuta-; o que la actividad que realizan estas mujeres era ilegal y preferían mantenerse al margen de esta problemática, es más, aconsejaron

\footnotetext{
${ }^{7}$ Se incluyó a una asociación fuera de la zona fronteriza que fue la Asociación Pro Derechos Humanos de Andalucía (APDHA) de Cádiz, debido a que esta era una organización que había trabajado activamente la situación de las porteadoras. También se entrevistó al tejido tercer sector de Melilla.

${ }^{8}$ Dentro de la Gendarmería marroquí este es uno de los puestos más demandados debido al sobresueldo en forma de "soborno" que reciben los agentes aduaneros, estimado por Seminario Independiente Al-Ayam por valor de 90 millones de euros anuales (APDHA, 2012).
}

REIM № 26 (junio 2019) ISSN: $1887-4460$ 
insistentemente a la investigadora que ella también se olvidase de este tema -esto aconteció exclusivamente en la wilaya de Tetuán-. Las entrevistas con las asociaciones se fijaron con el o la Presidenta, o en su defecto con el o la Coordinadora del área más relacionada con la situación de las mujeres porteadoras.

El resto de tercer sector y agentes sociales, se les fue contactando y fijando entrevistas durante las fases del trabajo de campo. En ocasiones algunas instituciones no contestaron a la propuesta como Autoridades Portuarias de Ceuta y las Cámaras de Comercio de Ceuta y Melilla; y en otros casos como los partidos políticos Caballas, PSOE de Ceuta y Centro Asesor de la Mujer de Ceuta (CAM), fue la incompatibilidad de agendas hizo imposible la entrevista.

La concertación de la entrevista se realizaba vía correo electrónico o por teléfono. En esa primera toma de contacto, se explicaba a la persona que iba a ser entrevistada el objetivo de la investigación y el interés que su participación tendría en el desarrollo de la tesis doctoral. En caso de que el contacto prosperase, se fijaba una cita para realizar la entrevista. Normalmente, la entrevista tenía lugar en las instalaciones del puesto de trabajo de la persona entrevistada, durante el horario matinal. A diferencia de las entrevistas con las mujeres porteadoras que todas se realizaron en su lengua materna, en el caso de los agentes sociales de la wilaya de Tetuán se realizaron entrevistas en castellano a quienes lo hablaban con fluidez; mientras que para quienes no dominaban el castellano, la entrevista se realizó en dariya, ${ }^{9}$ siguiendo el procedimiento de las entrevistas a las porteadoras descrito con anterioridad. La duración de las entrevistas era variable, desde los 30 minutos hasta la hora y media, dependiendo del tiempo que dispusiese la persona entrevistada y del conocimiento que tuviese sobre la materia objeto de investigación.

En total se entrevistó a 29 agentes sociales de la zona fronteriza, entre los que se encuentran miembros de partidos políticos, sindicatos, expertos de la temática, miembros de asociaciones y periodistas. Además de ello, en el proceso de saturación de las categorías emergió la necesidad de conocer la visión de las mujeres porteadoras sobre el tercer sector, con este propósito se entrevistó a 6 porteadoras en activo hasta que se saturaron estos códigos (Trinidad et al., 2006). En el proceso de entrevista a las mujeres porteadoras, se utilizó otro guion de entrevista en profundidad donde se incluyeron preguntas relativas a la opinión y relación de las porteadoras con el tercer sector.

Tabla 1: Agentes sociales entrevistados

\begin{tabular}{|l|c|}
\hline \multicolumn{1}{|c|}{ Cargo } & $\begin{array}{c}\text { Lugar de realización } \\
\text { de la entrevista }\end{array}$ \\
\hline Periodista en el Chamal Janoub & Tetuán \\
\hline Miembro de la Asociación Unión de la Acción Feminista & Martil \\
\hline Secretario General del SUP en Ceuta & Ceuta \\
\hline Responsable de proyectos de DIGMUN & Ceuta \\
\hline Secretario General de la Junta Sindical de TEX-Pec & Tetuán \\
\hline Área de Migración de la APDHA & Cádiz \\
\hline Periodista en el Chamal Post & Tetuán \\
\hline Delegado del Gobierno de Ceuta & Ceuta \\
\hline Presidente del Polígono de La Chimenea & Ceuta \\
\hline Área de Migraciones de Podemos Ceuta & Ceuta \\
\hline
\end{tabular}

${ }^{9}$ Es el dialecto del árabe que se habla en Marruecos. 


\begin{tabular}{|l|c|}
\hline Secretario General de la CECE & Ceuta \\
\hline Presidenta de la Asociación Nahda & Fnideq \\
\hline Miembro de la Asociación APISF & Tetuán \\
\hline $\begin{array}{l}\text { Presidente de la Asociación para la Cultura, Arte y Folclore } \\
\text { de Tánger }\end{array}$ & Tánger \\
\hline $\begin{array}{l}\text { Presidenta de la Asociación de empresarios de textil y } \\
\text { calzado reciclado de Ceuta }\end{array}$ & Ceuta \\
\hline Propietario de un comercio del Polígono del Tarajal \\
\hline $\begin{array}{l}\text { Trabajador de un establecimiento del polígono de La } \\
\text { Chimenea }\end{array}$ & Ceuta \\
\hline Presidenta de la Asociación Al-Ambar & Ceuta \\
\hline Secretario General de la AUGC en Ceuta & Ceuta \\
\hline Miembro de la Asociación Marroquí de Derechos Humanos & Ceuta \\
\hline Secretario General de la AUGC en Melilla & Tetuán \\
\hline $\begin{array}{l}\text { Presidenta de la Asociación del Desafío para el Desarrollo y } \\
\text { la Cultura }\end{array}$ & Melilla \\
\hline Secretario General del sindicato CGT en Melilla & Fnideq \\
\hline Coordinadora de la Fundación Cruz Blanca & Melilla \\
\hline Director de Tributos de Ceuta & Ceuta \\
\hline Profesora de l'Université Abdelmalek Essaâdi en Martil & Ceuta \\
\hline Profesor de la Universidad de Granada, campus Ceuta & Martil \\
\hline Portavoz en la Delegación de CC.Oo. en Ceuta & Ceuta \\
\hline Representante de la Asociación Tawaza & Martil \\
\hline
\end{tabular}

Fuente: Elaboración propia en base a los datos de la investigación.

Una vez realizada la entrevista, se completó una ficha en el diario de campo con los principales datos, traducción -en caso de ser necesaria-, transcripción y codificación de los datos.

La técnica de análisis de datos que se ha empleado ha sido la Teoría Fundamentada (Grounded Theory). El propósito de esta técnica o metodología de análisis es "Ilegar a conclusiones fundamentadas en los datos empíricos a través del llamado método comparativo constante (MCC), que permite una estrecha relación entre la recolección de datos, su análisis y teorización" (Sobczyk, 2015: 199-200). Siguiendo las características que exige esta metodología la fase de producción y de análisis de los datos se desarrolló de manera simultánea (Trinidad et al., 2006), de tal forma que una vez que se realizaba una entrevista, se transcribía y se codificaba. Este método facilitó el proceso emergencia y posterior saturación de las categorías. En esta investigación se empleó el muestreo teórico que cesa una vez se ha alcanzado la saturación teórica (Carrero et al., 2012), es decir, cuando los datos obtenidos de las entrevistas y de la observación comienzan a ser repetitivos. 


\section{Resultados}

El tejido asociativo de la región frontera es amplio, si se tiene en cuenta el número de asociaciones constituidas en Ceuta y en la wilaya de Tetuán. Únicamente en el municipio de Tetuán hay registradas 59 asociaciones, de las cuales el 32\% en sus estatutos recogen la defensa de los derechos humanos y de las mujeres, según el registro de asociaciones del Instituto Cervantes en Tetuán. En Ceuta, el número es idéntico que en Tetuán, son 58 asociaciones las que figuran como constituidas en el registro de asociaciones, pero el porcentaje de ellas que señala la defensa de los derechos humanos y de las mujeres es menor que Tetuán, con el $21 \%$ de las asociaciones dedicadas a esta finalidad. En el caso ceutí con un matiz, en este listado también se incluyen los partidos políticos y los sindicatos, es decir lo que se considera como tercer sector (Registro nacional de asociaciones y servicio de utilidad pública, 2016). Si bien se puede considerar que en la zona fronteriza una cuarta parte del tejido asociativo defiende los derechos humanos y de las mujeres, no hay ninguna asociación que tenga entre sus objetivos, ni en sus estatutos fundacionales, ni en su praxis, la lucha por la defensa de los derechos de las mujeres porteadoras.

"Durante este tiempo hemos estado indagando y nosotros trabajamos con muchas asociaciones, pero no hay ninguna asociación que trabaja con estas mujeres directamente o que trabajan exclusivamente. A partir del 92 del 93 surgieron un montón de asociaciones en Marruecos y realmente trabajar con, exclusivamente, con mujeres porteadoras no hay ninguna, no hay ninguna". (Amín Soussi, Área de Migración de la APDHA).

Como resultado del análisis cuantitativo del volumen y temáticas de tercer sector en la zona fronteriza, y especialmente, del análisis cualitativo de las entrevistas y de la observación, se han extraído tres puntos clave de familias de categorías ${ }^{10}$ que este artículo se estructuran en tres apartados de resultados: a) la confianza entre porteadoras y tejido asociativo; b) la postura del tejido asociativo ante la situación de las mujeres porteadoras; y c) el papel de los sindicatos en la problemática de las porteadoras.

\section{La escasa confianza de las porteadoras en el tejido asociativo de la zona fronteriza}

Las mujeres porteadoras tienen poca confianza en las asociaciones y son tienen la percepción de que el tejido asociativo ${ }^{11}$ no defiende sus derechos, al menos de manera directa. Si bien es cierto, que al estar constituidas asociaciones en la wilaya de Tetuán en defensa de los derechos de las mujeres que aglutinan objetivos como: la lucha contra de la violencia de género -en Marruecos se denomina violencia familiar- y la erradicación contra la pobreza femenina con especial atención a viudedad; las porteadoras acuden a las asociaciones por alguna de esas situaciones, no por la precariedad de su actividad laboral. Tanto es así que ocasiones optan por omitir que son

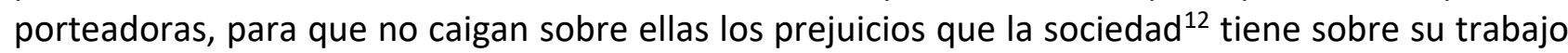
en la frontera.

\footnotetext{
10 Las familias de categorías que son asociaciones de códigos "que guardan relación entre sí, bien por el tema, el proceso, el tiempo, el grado de relación, las causas, las consecuencias etc.” (Carrero et al., 2012: 42).

${ }^{11} \mathrm{Al}$ comenzar la investigación se incluyó una pregunta sobre los sindicatos que se saturó rápidamente al no mostrar resultados que relacionen su actividad con la que realizan las porteadoras. Es por ello, que no se incorporan estos datos en este apartado.

12 Las porteadoras tienen un bajo prestigio social en la sociedad marroquí. La actividad laboral que realizan se considera ilegal en Marruecos -contrabando- y porque el modelo de vida que conlleva trabajar en la frontera, no está acorde con los valores tradicionales de la mujer marroquí (Aixelá, 2000).
} 
“No tenemos... bueno aunque tuviéramos, hay una asociación que te ayuda, que está en mi barriada, por si necesitas algo cuando estas enferma, tienes algún problema con el alquiler, no tienes con que comprar el borrego de la fiesta, pero si vas a quejarte y contarle sobre la frontera, te dicen tú eres rica, trabajas en la frontera, aunque les llores". (M'diq, 36 años, casada, tres hijos).

Las porteadoras que tienen contacto con asociaciones, principalmente de su barrio o cercanas a su casa, buscan la colaboración asistencialista del tejido asociativo, es decir, porque necesiten respaldo jurídico en el divorcio, apoyo psicológico o educativo para algunos de sus hijos, o con la colaboración del banco de alimentos con productos como la harina o el aceite.

“Nada, no existe nada de eso, todo eso es mentira, protocolo, no hay ni leyes ni nada, yo quería denunciar a mi marido, fui a la asociación, y nada aquí ni estoy casada ni divorciada, sentada aquí". (Casablanca, 46 años, casada, un hijo).

Las porteadoras no participan ni colaboran con las asociaciones de la wilaya de Tetuán y están alejadas de las actividades que organiza el tejido asociativo. La principal razón es que no tienen confianza en las asociaciones se puedan implicar en las problemáticas de las porteadoras, como ellas señalan: nada, no hay quien pregunte por ti. ${ }^{13}$ Tampoco las porteadoras han sido capaces de organizarse internamente para crear una comisión, asociación, colectivo o sindicato en el que ellas mismas defiendan sus derechos ante las autoridades de ambos lados y las proteja de las vulneraciones que sufren en el desempeño de su trabajo.

"No han llegado a formar una organización entre ellas. Formar una comisión ni tampoco asociación entre ellas... están abandonadas al destino, tratan de defenderse de buscar su método ilegal para hacer su trabajo. Las únicas manifestaciones, quejas que hubieron en los sitios fronterizos solamente es lanzar piedras conjuntamente como grupal pero no llegan a hablar, no llegaron a formar... ni tampoco a crear una vía legal para protestar, para reclamar sus derechos en ambas partes". (Mohssin Chergui, periodista del Chamal Janoub).

El periodista del diario tetuaní hace hincapié en que las porteadoras siguen utilizando métodos de protesta social poco organizados (Martínez-Bernal, 2015). Si bien se agrupan para una manifestación en la frontera internacional del lado marroquí para el lanzar piedras o palos de madera contra las autoridades porque cierren la frontera al paso de porteadoras, no son acciones coordinadas que repercutan en un movimiento posterior que aglutine los objetivos del colectivo de porteadoras (Quesada, 2012; Ramos, 1997). Son acciones concretas violentas de respuesta a la privación de poder realizar su trabajo ese día (Tarrow, 2004).

\section{El posicionamiento del tercer sector en la situación de las mujeres porteadoras}

Al analizar la posición y opinión del tercer sector sobre las porteadoras se dilucidan dos cuestiones claves. Por un lado, la percepción del tercer sector sobre la actividad que realizan las porteadoras, y por otro lado, la autovaloración de la implicación que tiene el tejido asociativo de la región fronteriza sobre la situación de estas mujeres.

El discurso del tercer sector sobre las porteadoras, tanto en Ceuta como en la wilaya de Tetuán, es paternalista. Son frecuentes las alusiones a la mala situación que sufren de las porteadoras con

${ }^{13}$ Código in vivo de la investigación en referencia a la implicación del tejido asociativo de la wilaya de Tetuán. 
expresiones como pobrecitas. Sin embargo, se experimenta una diferenciación sobre el contexto en el que el tercer sector aviva la actitud paternalista de las porteadoras (Alonso et al., 2011; Perreautl, 2004). En Ceuta se hace hincapié sobre las infraestructuras del circuito de las porteadoras y las condiciones de vida de Marruecos, que no dejan otra opción laboral a estas mujeres que trabajar como porteadoras.

“La pobreza en la que estas mujeres están inmersas es... es el problema fundamental, no se ve tanto, porque tú en la frontera lo que ves es la barbaridad de los bultos pero lo que hay detrás de eso es la pobreza en la que están inmersas. ¿Qué provoca esa pobreza? pues que viven en un país empobrecido... ¿qué provoca que un país este empobrecido? pues las desigualdades en el comercio internacional". (Paloma Manzano, Responsable de proyectos de DIGMUN).

Desde la wilaya tetuaní, el tercer sector se centra en las condiciones laborales de las porteadoras y en el grado de ilegalidad, legalidad o alegalidad del trabajo que realizan estas mujeres.

"No tienen medios, no tienen un trabajo digno dentro de Marruecos, no tienen Seguridad Social, no tienen paro... no tienen nada. La única ventana que tienen es esa y además si tienen hijos y el padre no está trabajando... con la pobreza de Marruecos la gente busca donde puede llevar su vida y si no hay nada legal entonces buscan cosas que son ilegales". (Abdelouahid Al Homade, miembro de la Asociación Marroquí de Derechos Humanos).

El acercamiento a la percepción que tiene el tejido asociativo sobre la actividad de las porteadoras es relevante para comprender el grado de implicación que ellos tienen sobre la situación de las porteadoras. La actitud generalizada versa sobre el reconocimiento propio de que no están a favor del trabajo que desempeñan estas mujeres. La causa que detectan desde ambos lados de la frontera, para no implicarse en la defensa de los derechos de estas mujeres es el difícil acceso a las porteadoras. Bien porque ellas mismas al entender su actividad como ilegal no se acercan a demandar ayuda al tejido social, o porque reniegan de su condición como porteadora, camuflando su actividad laboral por la de empleada doméstica en Ceuta.

“El problema es que como asociación tenemos muchas dificultades de acceso a estas mujeres, muchísimas eh... solamente estas mujeres han acudido a la asociación cuando han tenido problemas de violencia seria en la frontera (...) es un tema en el que no se trabaja mucho, desgraciadamente, trabajamos cuando hay una demanda por parte de las mujeres. No hay posibilidad de trabajar con estas mujeres". (Nadia Näir, miembro de la Asociación Unión de la Acción Feminista).

"Por nuestra experiencia no se trabaja con ellas porque no son accesibles y porque ellas van y vienen y no tienen tiempo para que tú hables con ellas de lo que necesitan". (Paloma Manzano, Responsable de proyectos de DIGMUN).

De tal forma que la relación entre porteadoras y tejido asociativo se encuentra bloqueada, puesto que las primeras no tienen confianza en que las asociaciones vayan a defender su situación y su problemática; y por parte de las asociaciones tienen la creencia de la dificultad y obstáculos de trabajar y colaborar con las porteadoras. Dicho escenario responde a una ausencia de comunicación real fluida entre porteadoras y el tejido asociativo de la zona fronteriza.

\section{El foco del sindicalismo sobre las porteadoras}

En virtud a los obstáculos que perciben las asociaciones de la zona fronteriza para defender los derechos de estas mujeres y la propia valoración de las porteadoras sobre que la actividad que realizan es ilegal, hay que sumarle que no se les otorga el estatus de trabajadoras. Desde el lado 
marroquí se las considera como contrabandistas, y desde la perspectiva ceutí son mujeres marroquíes que realizan compras a título individual en el polígono del Tarajal. Independientemente de la legalidad del fenómeno, es una consideración que sí que se tiene con el resto de transfronterizas -trabajadoras sexuales y empleadas domésticas- (Fuentes-Lara, 2016). Desde este punto de vista se abre un debate en torno a la funcionalidad de los sindicatos en la situación de las mujeres porteadoras.

“¿Qué estas no son trabajadoras? son trabajadoras, desempeñan un trabajo... y si los sindicatos realmente defienden el derecho de los trabajadores deberían tomar cartas en el asunto o decir algo por lo menos de... de estas mujeres tanto de un lado como en otro. $Y$ como he dicho antes, que es un tema que parece ser que nadie le importa". (Amín Soussi, Área de Migración de la APDHA).

En Ceuta, desde el sindicalismo no hay una defensa directa de la situación de las porteadoras. El sindicato Comisiones Obreras (CCOO) publicó en 2017 el informe "la problemática de los trabajadores transfronterizos" donde se hace referencia a las condiciones laborales de los transfronterizos, y tiene una actividad proactiva en la defensa de las condiciones laborales de las empleadas domésticas marroquíes en Ceuta. En cuanto a la cuestión de las porteadoras, el portavoz en la Delegación de CCOO en Ceuta, asegura que desde CCOO se ha abordado con los partidos políticos y con la Delegación de Gobierno la necesidad de proteger los derechos laborales de las porteadoras, sin llegar conseguir un compromiso en firme de las instituciones. El Delegado de CCOO en Ceuta también apunta que un factor a tener en consideración en la vulneración de derechos laborales de las mujeres porteadoras, y es el inexistente movimiento sindical marroquí. De igual modo, el sindicalismo marroquí reivindica que la actividad laboral que realizan las porteadoras se desarrolla en Ceuta, y por tanto son los sindicatos ceutíes y españoles quienes tienen más herramientas para proteger los intereses laborales de estas mujeres. A lo que se le añade la condición del porteo como una actividad ilegal en Marruecos, que tal y como señala, el Secretario General de la Junta Sindical de TEX-Pec, Mohamed Yasser es difícil englobar la actividad laboral que realizan las porteadoras para ser defendidas por los sindicatos marroquíes.

"El sindicato normalmente tiene un sector en el que trabaja, pero esa gente no tiene un sector donde puede trabajar, ¿dónde los clasificas a ellos? en la Federación Nacional de Porteadores o lo que sea... entonces están fuera de la ley".

La postura del sindicalismo marroquí es tajante con respecto a las porteadoras, al no ser consideradas como trabajadoras no pueden defender sus derechos, más aún cuando el propio sindicalismo califica la actividad laboral que realizan las porteadoras como ilegal y como causante de dañar la demanda interna y por ende sectores productivos marroquíes (Aziza, 2011).

La legalidad, ilegalidad o alegalidad del fenómeno no condiciona de manera drástica la conformación de un sindicato propio de porteadoras, al contrario de la opinión del Secretario General del sindicato marroquí de TEX-Pec, quién ironizaba sobre la creación de un sindicato de porteadoras. De hecho, la iniciativa del Sindicato Popular de Vendedores Ambulantes de Barcelona -conocidos como los manteros- son un ejemplo de cómo un grupo de personas extranjeras que realizan una actividad laboral de discutida legalidad, se constituyen como trabajadores y forman un sindicato para defender sus derechos laborales frente a la persecución policial y la criminalización social, como señala su portavoz Pape Diop (Iborra, 2016). Tras la pionera iniciativa en Barcelona, el Sindicato de Vendedores Ambulantes se ha constituido en Zaragoza, Valencia, Madrid y Granada. 
Por consiguiente, existen precedentes de iniciativas similares a la situación laboral de las porteadoras. Sin embargo, en el caso de estas mujeres los problemas que surgen son la autopercepción sobre que la actividad que realizan es ilegal y bajo prestigio social de su profesión, a lo que se le suma el temor a las represalias por parte de la policía. De tal modo, que si empiezan a organizarse prohíban el porteo, o les restrinjan la entrada a Ceuta a los miembros de la organización. Estas vulnerabilidades las refleja Mohssin Chergui, periodista del Chamal Janoub:

"Aunque sean trabajadoras se las tratan como inmigrantes ilegales".

\section{Conclusiones}

La situación de las mujeres porteadoras en la frontera ceutí responde a un fenómeno multicausal en el cual hay múltiples agentes involucrados. El sistema que siguen los bienes y mercancías desde que desembarcan en el puerto de Ceuta hasta que llegan al lado marroquí de la frontera es férreo y bien estructurado. Tal y como se ha dicho con anterioridad el comercio atípico no es una actividad residual sino que es uno de los pilares -sino el principal- sobre el que se sustenta la economía ceutí. Sin embargo, en toda la estructuración del comercio atípico son las mujeres porteadoras quienes son el eslabón más vulnerable del circuito de la mercancía. Ellas son quienes reciben el mayor desgaste sobre su cuerpo en el desarrollo de su trabajo -cargar con el fardo, las agresiones físicas de la policía, deterioro físico, fallecimiento ${ }^{14}$-, y psicológico -maltrato verbal de comerciantes y policía, presión social en su sociedad de origen-.

Ante tal escenario resulta significativo que el gobierno español y la Unión Europea no generen soluciones reales para mejorar la situación de las mujeres porteadoras, pero aún más conmoción provoca que el tejido asociativo de la zona fronteriza no se involucre en defender los derechos de las porteadoras. Justamente, un tejido asociativo organizado y activo en la defensa de los derechos humanos de personas en tránsito migrante -Asociación Elín en Ceuta o Manos Solidarias en Tetuán-; con las trabajadoras sexuales -Fundación Cruz Blanca en Ceuta-; con empleadas domésticas o del hogar-Asociación DIGMUN en Ceuta-; o con la violencia de género -Asociación Tawaza en Martil-.

La explicación, como señalan las propias asociaciones, se puede deber a la dificultad para acceder a las porteadoras, la consideración social de que la actividad que realizan es ilegal, que no se les considere como trabajadoras o la falta de organización interna. Sin embargo, parece responder a la visión paternalista que se tiene de las mujeres porteadoras, lo cual provoca que el tejido asociativo las analice como sujetos sin derechos que reciben un importe económico por realizar una actividad laboral ilegal. Esta misma consideración no se ha tenido por parte de la opinión pública y del tejido asociativo para la situación de los manteros ni de las personas en tránsito migrante. Ante esto cabe preguntarse si también habrá un sesgo patriarcal que invisibilice, aún más, los derechos humanos de las mujeres porteadoras.

Una alternativa para la defensa de los derechos de las mujeres porteadoras desde el tejido asociativo sería una coalición de cooperación transnacional entre la zona fronteriza. La diferencia entre la red y la coalición trasnacional de asociaciones es que es la segunda es una organización más formal y donde el tejido social integrante adquiere mayores compromisos ante el grupo o la finalidad que defienden (Avendaño, et al., 2000). La utilidad de constituir una coalición trasnacional es que el tejido social de ambas zonas fronterizas ante situaciones de conflicto o de violación fragante de derechos humanos se reivindique de manera conjunta, y con unos objetivos

\footnotetext{
${ }^{14}$ Desde la apertura del nuevo paso fronterizo entre Ceuta y Marruecos, el Tarajal II, el 27 de febrero de 2017, han muerto al menos, en el desempeño de su trabajo 7 mujeres, que se suman a los fallecimientos de Bushra y Zhora en mayo de 2009 dentro del polígono del Tarajal.
} 
previamente constituidos, que puedan llegar a generar algún avance en la situación de las porteadoras. Esta coalición la formaría el tejido asociativo de la región transfronteriza que esté relacionado con la temática de las mujeres porteadoras, es decir, asociaciones en defensa de las mujeres, de la violencia de género, contra la pobreza social y femenina, de respecto a los derechos humanos etc. que además y según los datos de asociacionismo de la región fronteriza, ya habría una base férrea constituida que conforman una cuarta parte del tercer sector de Ceuta y de Tetuán.

La ventaja que otorga una coalición transfronteriza de asociaciones es crear un grupo de presión unido en defensa de las vulnerabilidades de la región transfronteriza que por un lado genere influencia en las autoridades de ambos lados y por el otro, cubra las deficiencias que las instituciones gubernamentales de la región fronteriza no protegen o no actúan (De la 0,2008 ) en esta problemática. En la frontera entre México y Estados Unidos, existen ejemplos similares de coalición transfronteriza de asociaciones. Las coaliciones más antiguas se registran en la Baja California como apoyo a los trabajadores migrantes, se constituyeron como el Centro de Investigaciones y Estudios Migratorios (CIEM) y en el Centro Binacional de Derechos Humanos (CBDH) (Avendaño et al., 2000).

Un caso similar al de las mujeres porteadoras es el ejemplo de la defensa de los derechos laborales de las mujeres de Kukdong -que posteriormente pasó a llamarse Mexmode-. Las trabajadoras de la maquila de la empresa coreana Kukdong International establecida en Atlixco, Puebla (México), consiguieron que asociaciones, ONG internacionales y sindicatos, tanto de México como de Estados Unidos, se implicarán en su lucha por unos derechos laborales justo y conformarán una coalición transnacional. Los resultados de esta coalición significaron la dignificación de las condiciones laborales de las maquiladoras, que posteriormente y en virtud a la experiencia adquirida, crearon su propio sindicato, el Sindicato Independiente de Trabajadores de la Empresa Mexmode (De la O, 2008) o desde el mero sindicalismo como el caso del Sindicato de Vendedores Ambulantes, conocidos popularmente como manteros.

\section{Referencias}

ALONSO, Isabel; MCCABE, Anne y CHORNET, Daniel (2011): 'En sus propias palabras: la construcción de la imagen del inmigrante en la prensa española', Discurso \& Sociedad, no 5, pp. 547-568.

APDHA (2012). Declaración de Tetuán, Sevilla, Asociación Pro Derechos Humanos de Andalucía. AIXELÁ, Yolanda (2000). Mujeres en Marruecos. Un análisis desde el parentesco y el género. Barcelona: Edicions Bellaterra.

AVENDAÑO, Rosa María; MORENO, José A. y PRIEGO, Enrique (2000): 'Las ONG en la diplomacia ciudadana', Estudios Fronterizos, no 1, pp. 89-135.

AZIZA, Mimoun (2011). Une frontière européenne en terre marocaine. Analyse des relations transfrontalières entre Nador et Melilla. En Ribas-Mateos, Natalia (ed.), El río bravo Mediterráneo. Las regiones fronterizas en la época de la globalización (pp. 307-320). Barcelona, Bellaterra.

BOLETÍN OFICIAL DEL ESTADO (BOE) no 81, de 5 de abril de 1994 de Acuerdo de Adhesión del Reino de España al Convenio de aplicación del Acuerdo de Schengen de 14 de junio de 1985 entre los Gobiernos de los Estados de la Unión Económica Benelux de la República Federal de Alemania y de la República Francesa [en línea]. Disponible en: https://www.boe.es/boe/dias/1994/04/05/pdfs/A10390-10422.pdf [Consulta: 2018, 26 de junio]. 
CARABAZA, Enrique y DE SANTOS, Máximo (1993): Melilla y Ceuta. Las últimas colonias, Madrid, Talasa Ediciones.

CARRERO, Virginia; SORIANO-MIRAS, Rosa; y TRINIDAD, Antonio (2012): Teoría Fundamentada Grounded Theory. El desarrollo de teoría desde la generalización conceptual, Madrid, Cuadernos Metodológicos, no 37, 2a edición revisada. Centro de Investigaciones Sociológicas (CIS).

COMISIONES OBRERAS (CCOO) (2017). La problemática de los trabajadores transfronterizos. Infoservicios. Disponible en: https://www.ccoo-servicios.info/noticias/pdf/35998.pdf [Consulta: 2019, 6 de enero].

DÍAZ DE RADA, Ángel (2011): El taller del etnógrafo. Materiales y herramientas de investigación en Etnografía, Madrid, UNED.

DE LA O, María (2008): 'Las mujeres y los movimientos de defensa laboral entre México y Estados Unidos: un análisis de su influencia en comunidades no fronterizas', Estudios Políticos, no 32, pp. 255-275.

ESPIÑEIRA, Keina (2013): 'Colonialidad en la frontera Ceutí. Sebta mashi Schengen, mashi Euroba', Ecléctica, Revista de estudios culturales, no 2, pp. 5-18.

FERRER-GALLARDO, Xavier (2008): 'Acrobacias fronterizas en Ceuta y Melilla: explorando los perímetros terrestres de la Unión Europea en el continente africano', Documents d'Anàlisi Geogràfica, no 51, pp. 129-149.

FERRER-GALLARDO, Xavier y ALBET-MAS, Abel (2016). 'EU-Limboscapes: Ceuta and the proliferation of migrant detention spaces across the European Union', European Urban and Regional Studies, no 23, pp. 527-530. https://doi.org/10.1177/0969776413508766

FUENTES-LARA, M. Cristina (2016): 'El comercio atípico en la frontera hispano-marroquí. El caso de las porteadoras', Revista Internacional de Estudios Migratorios, no 6, pp. 84-107.

FUENTES-LARA, M. Cristina (2017a): 'Carrier' women: a Grounded Theory approach', Revista Internacional de Estudios Migratorios, no 7 (3), pp. 153-175.

FUENTES-LARA, M. Cristina (2017b): 'Ceuta y su hinterland desde la teoría de la frontera. El caso de las mujeres porteadoras'. Revista de Paz y Conflictos, no 10 (2), pp. 165-185.

FUENTES-LARA, M. Cristina (2017c): La situación de las mujeres porteadoras en la frontera hispano-marroquí. El caso de Ceuta, Granada, Universidad de Granada.

FUENTES-LARA, M. Cristina (2018): 'Las mujeres porteadoras y el comercio irregular en la frontera de Ceuta', en FERRER-GALLARDO, Xavier y GABRIELLI, Lorenzo (ed.): Estados de excepción en la excepción del Estado: Ceuta y Melilla.

GALÁN, Nuria (2012). Mujeres transfronterizas: marroquíes empleadas del hogar en Ceuta. Tesis Doctoral, Granada, Universidad de Granada.

GARCIA-FLORÉZ, Dionisio (1999): Ceuta y Melilla. Cuestión de Estado, Melilla, Ciudad Autónoma de Melilla.

GONZÁLEZ, Virgilio y LÓPEZ-GUZMÁN, Tomás (2009): 'Melilla: fiscalidad local y actividad comercial. Una reflexión', Boletín económico de ICE, no 2958, pp. 37-43.

IBORRA, Yeray (08/10/2016): El Sindicato de manteros crea su propia cooperativa para seguir luchando en la calle. Eldiario.es.

LÓPEZ-BUENO, José María (2008): Melilla, una oportunidad para las relaciones hispanomarroquies. Un modelo al amparo de la Política Europea de Vecindad, Madrid, Ediciones Académicas.

LÓPEZ-SALA, Ana M. (2012): 'Donde el sur confluye con el norte: movimientos migratorios, dinámica económica y seguridad en las relaciones bilaterales entre España y Marruecos', Documentos CIDOB. Migraciones, no 24, pp. 1-12. 
MARTíN, José Aureliano (2010): 'Una propuesta metodológica para estimar el impacto del desarme arancelario de Marruecos sobre la Economía de Ceuta', en GÓMEZ, Cecilio (coord.): Estudios sobre el Régimen Económico y Fiscal de Ceuta: Presente y futuro, Granada, Centro de Estudios Jurídicos Granada, pp. 139-160.

MARTÍNEZ-BERNAL, Daniel R. (2015): Caminando hacia la Satyagraha. Comunidad, lucha y Kweer Fxindxi en los Nasa (1971-2015), Granada, Universidad de Granada.

MOFFETTE, David (2013): 'Muslim ceutíes, migrants, and porteadores: Race, security, and tolerance at the Spanish-Moroccan border', Canadian Journal of Sociology, no 38, pp. 601-621.

MORÉ, Iñigo (2007): La vida en la frontera, Madrid, Marcial Pons.

MUÑOZ, José (1986): La fiscalidad de los territorios de Ceuta y Melilla, Melilla, Universidad Nacional de Educación a Distancia.

ORTÍ, Alfonso (2010): 'La apertura y el enfoque cualitativo o estructural: la entrevista abierta semidirectiva y la discusión de grupo', en GARCÍA-FERRANDO, Manuel; IBÁÑEZ, Jesús y ALVIRA, Fernando (eds.): El análisis de la realidad social. Métodos y técnicas de investigación, Madrid, Alianza Editorial, pp. 219-282.

PÉREZ-DÍAZ, Víctor y LÓPEZ-NOVO, Joaquín (2003). El tercer sector social en España, Madrid, Ministerio de Trabajo y Asuntos Sociales.

PERREAULT, Samuel (2004): 'Visible minorities and victimization', Canadian Centre for Justicie Statistics Profile Series, № 15, pp. 1-18.

PLANET CONTRERAS, Ana Isabel (1998): Melilla y Ceuta. Espacios-frontera. Hispano-marroquíes, Melilla, Ciudad Autonóma de Melilla, Ciudad Autonóma de Ceuta y UNED-Melilla.

PRESUPUESTOS CIUDAD AUTÓNOMA DE CEUTA (2017): Documentos. Secciones. Presupuestos. Ciudad Autónoma de Ceuta.

QUESADA, Encarnación (2012): 'La perspectiva de género en el comercio justo: una reflexión económico-antropológica', Revista pueblos y fronteras digital, no 14, pp. 81-116.

RAMOS, Luisa (1997): 'La dimensión política de los movimientos sociales: Algunos problemas conceptuales', Reis, no 79, pp. 247-263.

ROSADO-CARO, Ana M. y FUENTES-LARA, M. Cristina (2018): 'Las invisibles tras el fardo de nuestra vergüenza: las porteadoras en la frontera sur de Europa', en ROSADO-CARO, Ana M. y LARA, Rafael (coord.): Derechos humanos en la frontera sur, Sevilla, APDHA, pp. 81-92.

RUIZ-OLABUÉNAGA, José Ignacio (1996): Metodología de la investigación cualitativa, Bilbao, Universidad de Deusto.

SACEDA, Marta (2016): Evolución de las migraciones entre Marruecos y España (1999-2014): la gestión migratoria en Ceuta ante la encrucijada de intereses UE-Marruecos, Madrid, Universidad Complutense de Madrid.

SOBCZYK, Rita (2015): Diversidad religiosa en el contexto migratorio andaluz: identidad, fronteras sociales y religión "vivida", Granada, Universidad de Granada.

TARROW, Sidney (2004): 'Bridging the quantitative-qualitative divide', en BRADY, Henry y COLLIER, David (eds.): Rethinking Social Inquiry, Diverse Tools, Shared Standards, Maryland, Rowman \& Littlefield Publishers, pp. 171-180.

TRINIDAD, Antonio, CARRERO, Virginia y SORIANO-MIRAS, Rosa (2006): Teoría fundamentada "Grounded Theory". La construcción de la teoría a través del análisis interpretacional, Madrid, Cuadernos Metodológicos, no 37, Centro de Investigaciones Sociológicas (CIS).

VELASCO, Honorio y DÍAZ DE RADA, Ángel (2006): La lógica de la investigación etnográfica. Un modelo de trabajo para etnógrafos de la escuela, Madrid, Trotta. 